\title{
The intravesical injection of highly purified botulinum toxin for the treatment of neurogenic detrusor overactivity
}

Denise Asafu-Adjei ${ }^{1}$; Alexander Small²; Glen McWilliams ${ }^{3}$; Giuseppe Galea ${ }^{4}$; Doreen Chung; Jamie Pak ${ }^{1}$

${ }^{1}$ Columbia University Medical Center, New York, NY, United States; ${ }^{2}$ Department of Urology, Columbia, New York, NY, United States; ${ }^{3}$ James J. Peters Veterans Affairs Medical Center University, New York, NY, United States; ${ }^{4}$ New York Presbyterian Hospital Brooklyn Methodist Hospital, New York, NY, United States

Cite as: Can Urol Assoc J 2020 May 12; Epub ahead of print. http://dx.doi.org/10.5489/cuaj.6182

Published online May 12, 2020

$* * *$

\section{Abstract}

Introduction: We aimed to assess safety and efficacy of incobotulinumtoxinA for the treatment of neurogenic detrusor overactivity (DO).

Methods: We identified patients with neurogenic DO confirmed on urodynamics (UDS) and reported urgency incontinence (UI) in those who received intravesical incobotulinumtoxin A injection for neurogenic bladder between November 2013 and May 2017. Parameters studied were daytime frequency, daily incontinence episodes, daily pad use, clean intermittent catheterization (CIC) volumes, symptom scores (UDI6, IIQ7, PGII), and complications.

Results: We examined 17 male patients who met inclusion criteria and underwent incobotulinumtoxinA injection. Mean age was $61.2 \pm 15.4$ years. Fourteen patients $(82 \%)$ were taking oral antimuscarinics prior to the incobotulinumtoxin A injection. There were improvements in the following parameters: average daily pads ( 4.5 to $3.3, \mathrm{p}=0.465$ ), daily urinary frequency $(9.4$ to $4.6, p=0.048)$, daily incontinent episodes $(2.5$ to $0.4, p=0.033)$, CIC volumes ( 400 to $550 \mathrm{~mL}, \mathrm{p}=0.356$ ), hours in between CIC (3.6 to $5.2, \mathrm{p}=0.127$ ), and the validated questionnaires UDI6 (30.6 to 7.4, $\mathrm{p}=0.543$ ) and IIQ7 (52.4 to 6.8, $\mathrm{p}=0.029$ ). There were no documented symptomatic urinary tract infections (UTIs) within 30 days of injection or reports of de novo urinary retention. Nine of 17 patients $(53 \%)$ reported being dry at their first postoperative visit.

Conclusions: In this preliminary pilot study of a small cohort of males with neurogenic DO and UI, significant improvements were seen following incobotulinumtoxinA injection in daily frequency, incontinence episodes, hours in between CIC, and quality of 
life. Larger-scale and long-term studies are required to confirm these results, but initial findings are promising for wider use of this formulation.

\section{Introduction}

Intradetrusor injection of botulinum toxin type $A$ is an effective treatment for overactive bladder (OAB) and neurogenic detrusor overactivity (NDO) ${ }^{1}$. Botulinum toxin $\mathrm{A}$ is one of seven serotypes of toxins produced by Clostridium botulinum. There are several commercial forms of botulinum toxin A, all with similar toxin structures, but with slightly different protein modifications ${ }^{2}$. One commercial form of botulinumtoxinA, onabotulinumtoxin $A$ or Botox $\AA$, is FDA approved for urinary incontinence (UI) and overactive bladder (OAB) symptoms ${ }^{3}$. Multiple pivotal studies have shown the effectiveness of onabotulinumtoxin $\mathrm{A}$ in idiopathic overactive bladder syndrome $(\mathrm{OAB})^{4}$, ${ }^{5}$. In patients with NDO, multiple trials have also demonstrated effectiveness of onabotulinumtoxinA for urinary incontinence. In a phase III, double-blind, randomized, multicenter placebo controlled trial by Ginsberg et al, 416 patients with spinal cord injuries or multiple sclerosis were randomized to either onabotulinumtoxinA or placebo ${ }^{6}$. OnabotulinumtoxinA significantly reduced UI episodes/week and improved urodynamic parameters and quality of life compared to placebo in patients with $\mathrm{NDO}^{6}$. In an extension study benefit was still seen at 4 years with repeat treatments as needed ${ }^{7}$ AbobotulinumtoxinA (Dysport ${ }^{\circledR}$ ) and rimabotulinumtoxinB $\left(\right.$ NeuroBloc $\left.{ }^{\circledR}\right)$ are other neurotoxin forms available in the U.S. that have been studied for use in the bladder. In a prospective study of 207 patients with idiopathic OAB by Ravindra et al, abobotulinumtoxinA was found to be inferior to onabotulinumtoxinA for the treatment of idiopathic $\mathrm{OAB}$ as it had twice the rate of symptomatic urinary retention requiring $\mathrm{CIC}^{8}$. It was shown these toxins were not interchangeable at equal doses ${ }^{8}$. Peyronnet et al. reported on the efficacy and safety of intradetrusor injection of abobotulinum toxin $\mathrm{A}$ $750 \mathrm{U}$ in patients with neurogenic $\mathrm{DO}^{9}$. The success rate, which was a composite parameter including continence, number of catheterizations, and lack of DO, was 64.2. In 2007, Hirst et al. studied the effect of intradetrusor rimabotulinumtoxinB in a nonrandomized study including 25 patients with both idiopathic and neurogenic detrusor activity (5 with NDO due to transverse myelitis and multiple sclerosis). Subjective improvements were seen in a small portion of the idiopathic detrusor activity patients, with two NDO patients reporting no improvement ${ }^{10}$. This study showed a short duration of action of rimabotulinumtoxinB, with effects wearing off by 10 weeks in most of their patients $^{10}$.

IncobotulinumtoxinA, commercially known as Xeomin ${ }^{\circledR}$ (Merz Pharmaceuticals $\mathrm{GmbH}$, Frankfurt, Germany), is another commercially available form of botulinumtoxinA 
and was approved by the FDA in 2011 for cervical dystonia and blepharospasm ${ }^{11}$. It is a highly purified form of this toxin with no complexing proteins ${ }^{11}$. It has been postulated that given its purity, there is less immunogenicity in patients. Potentially decreased immunogenicity of this botulinumtoxinA may be particularly advantageous in the neurogenic population, who often receive injections in multiple different organs. However, it is unclear if the differences in immunogenicity are clinically relevant in this setting. There are no maximum dosing ranges published by the manufacturer for the intravesical administration of incobotulinumtoxin. For limb spasticity incobotulinum toxin has shown to be as effective as ONA with a comparable adverse event profile when a clinical conversion ratio of $1: 1$ was used $^{12}$. In limb spasticity, dosage of up to $800 \mathrm{U}$ have been used without compromising safety or tolerability ${ }^{13}$.

In contrast to onabotulinum toxin, incobotulinumtoxin can be stored at room temperature for $\leq 36$ months before reconstitution. There is also evidence that equal product efficacy with $25^{\circ} \mathrm{C}$ storage (room temperature) can be maintained 1 week post reconstitution ${ }^{14}$. Incobotulinumtoxin $A$ and onabotulinutoxinaA were found to have comparable effects on DO and similar adverse event profiles with an almost 1:1 conversion ratio ${ }^{15}{ }^{16}$. Most studies on NDO outcomes have involved Dysport ${ }^{\circledR}$ and Botox ${ }^{\circledR}{ }^{17}$. Dosing differences and interchangeability between toxin types in terms of clinical impact remains an important area of future research ${ }^{17}$.

Additionally, incobotulinumtoxinA is generally less expensive than onabotulinumtoxin $\mathrm{A}$, making this an attractive and more cost-friendly alternative for OAB patients with UI. Tilden et al showed that incobotulinumtoxinA was more cost effective, compared to onabotulinumtoxinA in patients with blepharospasm and cervical dystonia in the Australian health care system ${ }^{18}$. They found an incremental cost/qualityadjusted life-year gained of $\$ 25,588$ in incobotulinumtoxin $A$ and $\$ 23,794$ in onabotulinumtoxin $\mathrm{A}^{18}$. The effectiveness of this more purified, less immunogenic incobotulinumtoxinA for neurogenic DO with UI has not yet been well studied.

In 2015, incobotulinumtoxinA was approved in the U.S. for upper limb spasticity in adults. Other non-FDA indications include chronic sialorrhea and temporary improvement in the appearance of moderate to severe glabellar lines with corrugator and/or procerus muscle activity ${ }^{19}$. Some benefits of incobotulinumtoxinA versus placebo for upper limb spasticity were shown in the post-stroke population by Kanovsky et al in a prospective, non-randomized study of 148 people, completed in 23 European sites ${ }^{20}$. IncobotulinumtoxinA injections resulted in significant and sustained improvements in muscle tone and disability in this patient population ${ }^{20}$.

To date, this formulation has not yet been well studied for use in the bladder. In a study by Conte et al., the objective was to investigate whether bladder dysfunction in MS patients depends on suprapontine or spinal lesions. $25 \mathrm{MS}$ patients who were spontaneously voiding and had low PVRs underwent intradetrusor injection with 100U 
incobotulinumtoxinA. ${ }^{21}$. No significant differences were seen in the Hoffman reflex after injection. However, there were improvements in volumes at first, normal, strong desire, dyssynergic activity, and voiding diary parameters ${ }^{21}$.

The primary objective of this study was to assess the safety and effectiveness of incobotulinumtoxinA in patients with neurogenic DO and UI.

\section{Methods}

\section{Study design and participants}

After Institutional Review Board (IRB) approval, patients were identified in a retrospective manner at a single Veteran's Affairs Medical Center (VAMC) with neurogenic bladder who had undergone intradetrusor incobotulinumtoxinA injections between November 2013 and May 2017. All patients had previously failed antimuscarinic therapy and had neurogenic DO and UI. There was no strict definition utilized to define antimuscarinic failure. It was assumed that patients who had persistent bothersome voiding symptoms while on the medical therapy were refractory. All patients underwent detailed history, physical examination, urinalysis, urine culture, and video urodynamics (UDS) prior to injection.

Video UDS were done according to International Continence Society (ICS) standards with fluid filled system and external transducers at the reference level of the upper edge of the symphysis pubis using Medtronic ${ }^{\circledR}$ UDS equipment ${ }^{22}$. The baseline bladder capacities of all patients were determined from the most recent UDS preceding the first injection of incobotulinumtoxinA.

Patients with injections of onabotulinumtoxinA within 1 year of incobotulinumtoxinA treatment were excluded. Of note, patients with prior onabotulinumtoxinA injections were receiving incobotulinumtoxinA due to the new hospital standard of administering this less expensive formulation. However, there was varying levels of efficacy and satisfaction with the onabotulinumtoxinA among the patients. We recorded demographic patient information and baseline clinical data. Additionally, the patients were assessed for average voiding parameters at home, including daily pad use, frequency, incontinence episodes, and CIC volumes. Patient responses to the following validated questionnaires were also recorded: Incontinence Impact Questionnaire (IIQ-7), Urogenital Distress Inventory (UDI-6), and Patient Global Improvement of Incontinence (PGI-I). The averages of the IIQ-7 and UDI-6 surveys were converted to a 100-point scale. While there are no globally accepted cutoffs, higher scores on the 100 point scale represent higher impact and distress scores ${ }^{23}$. Patients returned for a follow-up visit within 2-4 weeks following the incobotulinumtoxinA injection and all patients were evaluated. Complications within 30 days of incobotulinumtoxinA injection and de novo urinary retention were recorded. 


\section{Statistical analyses}

Chi-squared and Fisher's Exact test was used for categorical variables and the MannWhitney $\mathrm{U}$ test for continuous variables. All data analyses were done using IBM SPSS software, Version 23 (IBM Corporation, Armonk, NY). Multivariate analysis was attempted, but limited due to the small sample size. Statistical analysis was done with SPSS Statistics 23.0.

\section{Procedure technique}

Patients with positive urine cultures were treated with appropriate antibiotics prior to injection. Patients were placed in the dorsal lithotomy position and administered either local anesthesia or conscious sedation, depending on patient anxiety level and/or history of autonomic dysreflexia from other local procedures. Incobotulinum toxin was diluted in normal saline, with $200 \mathrm{U}$ diluted in $20 \mathrm{~mL}$ and $300 \mathrm{U}$ diluted in $30 \mathrm{~mL}$. Given this was a retrospective study with a heterogeneous group of urologists; the amount of incobotulinumtoxin administered was at the discretion of the respective providers. A rigid cystoscope was inserted through the urethra under direct vision and $1 \mathrm{~mL}$ injections were distributed throughout the bladder wall including at the trigone.

\section{Results}

\section{Patient demographics and baseline characteristics}

A total of 17 participants with neurogenic DO (confirmed on UDS) and refractory UI were included in this study. All participants were male with mean age of $61.2 \pm 15.4$ years. Prior to incobotulinumtoxin injection, six (35\%) patients had indwelling Foley, six (35\%) were voiding on their own, and five $(30 \%)$ were doing CIC. Eleven patients had SCI: four thoracic spine injuries and seven cervical spinal cord injuries. One patient had a primary diagnosis of multiple sclerosis (MS) with a history of stroke. Two patients had diagnoses of Parkinson's disease (one with a concurrent thoracic SCI) and four had a history of stroke (with one also having MS). 5 (29\%) patients overall had a concurrent diagnosis of severe traumatic brain injury (TBI).

Two (12\%) patients had diabetes mellitus (DM) and $3(18 \%)$ patients had a history of chronic kidney disease (CKD). Table 1 summarizes baseline characteristics of the participants. Comorbidities were captured with the Charlson Age Comorbidity indexes, reported in Table 1. In terms of previous conservative management, $82 \%$ of the participants had been using oral antimuscarinics regularly for at least one month prior to their first incobotulinumtoxinA injection.

Eight (47\%) participants had undergone previous injections with onobotulinumtoxinA. (greater than one year before incobotulinumtoxinA injection). Nine (53\%) patients had repeat injections of incobotulinumtoxinA after their initial injection in 
the study period. Seven (41\%) participants had injections with 300 units and ten patients $(59 \%)$ received 200 units.

Overall, $100 \%$ of patients underwent repeat intradetrusor incobotulinum toxin within 1 year of the initial procedure. Median time to first repeat injection was 34 weeks (IQR 29-64). 24\% of patients underwent more than one repeat injection within 1 year of the initial study injection.

\section{Clinical outcomes}

Postoperative data was obtained from two weeks to one month after the patients' first incobotulinumtoxinA injection. Table 2 illustrates outcomes following incobotulinumtoxinA injection in patients who were already on a CIC regimen. Following treatment, the median for daily pads was stable at 0 . The median number of incontinent episodes decreased from 2 to 0 after treatment. Median volumes with CIC improved, 400 to 550, as well as median number of hours between CIC, 4 to 6 pre and post-treatment. Average scores for the UDI6 and IIQ7 surveys also improved pre and post-treatment, respectively. All patients in this group were dry following their initial injection, with no one requiring pads after treatment.

Table 3 outlines similar outcomes in patients who were voiding prior to this study. Following treatment, the median for daily pads was 12 from 7.5. The median number of incontinent episodes decreased from 2.5 to 1 after treatment. Average scores for the UDI6 and IIQ7 surveys also improved pre and post-treatment, respectively. Two patients versus none were dry after treatment. All patients in this group were dry following their initial injection, with no one requiring pads after treatment.

Finally, Table 4 summarizes results for the subgroup with chronic Foley catheters and SP tubes. Creatinine levels remained stable over time. Average IIQ7 and UDI-6 survey scores were also markedly improved in this group. All patients were dry after an initial course of treatment.

Ten participants overall completed PGI-I surveys postoperatively and results are shown in Figure 1. In the non-voiding group, three participants completed the survey. One participant each (33\%) endorsed feeling a little better, much better, and very much better, totaling $\sim 33 \%$ for each response. In the voiding group, four participants $(66.6 \%)$ endorsed feeling very much better after their initial incobotulinumtoxinA injection. One participant each (16.7\%) endorsed feeling a little better and much better. Of note, no participants reported no improvement or feeling worse after treatment. Nine of the seventeen patients $(53 \%)$ reported being dry at their first postoperative visit. One patient who initially had an indwelling Foley prior to incobotulinumtoxinA treatment was able to successfully initiate and perform CIC following intravesical treatment. This was a patient with bilateral Grade 4 vesicoureteral reflux and a small bladder capacity of about $215 \mathrm{~mL}$ on UDS. Of note, this patient had resolution of hydronephrosis on renal ultrasound following intravesical treatment with incobotulinumtoxin. 


\section{Incobotulinumtoxin A to treat neurogenic detrusor overactivity}

No patients had documented symptomatic urinary tract infections (UTIs) or other adverse effects in the 30 days following treatment.

\section{Discussion}

In this cohort of patients with neurogenic bladder and DO there were clinically significant improvements in frequency and daily incontinent episodes after an initial injection with incobotulinumtoxinA. Additionally, in patients doing CIC, significant improvements were seen in time between catheterization. Furthermore significant improvements were seen in quality of life scores. All patients, whether voiding or not, reported global impression of improvement following treatment. The initial results of this pilot study provide promising evidence that incobotulinumtoxin A can be a reasonable alternative to onabotulinumtoxin $\mathrm{A}$ in patients with $\mathrm{UI}$ and other $\mathrm{OAB}$ symptoms.

To our knowledge, this is the first study of its kind to examine the effects of incobotulinumtoxinA on bladder diary, urodynamic parameters and quality of life measures in a range of patients with NDO, both voiding and not voiding. Only one other study has examined the effect of intradetrusor injection of incobotulinumtoxinA. Conte et al. completed a study investigating the neurophysiological effects of $100 \mathrm{U}$ incobotulinumtoxinA in patients with neurogenic DO due to multiple sclerosis who were spontaneously voiding with low post-void residual ${ }^{21}$. The primary aim of the study was to investigate whether in MS bladder dysfunction is due to suprapontine or spinal lesions, by examining change in the soleus Hoffman reflex. This study showed improvements in first, normal, and strong desire during UDS, with no change in the Hoffman reflex ${ }^{21}$. This population of neurogenic bladder patients was very different than our patient population, comprised mostly of SCI patients of which only $35 \%$ were voiding spontaneously. Furthermore, the dosage of medication used was different.

Our results are very similar to those observed in patients with neurogenic DO who have undergone intradetrusor injection of onabotulinumtoxinA. Cruz et al. randomized patients with MS (n-154) or SCI $(n=121)$ to placebo, 200U onabotulinumtoxinA, of 300U onabotulinumtoxin A. At week 6, significant improvements were seen compared to placebo in incontinence episodes per week, urodynamics parameters and quality of life.

This is the first paper to look at voiding parameters after any botulinumtoxin formulation in the bladder of TBI patients, despite neurogenic bladders being common sequelae of TBI. Prior literature has studied outcomes of botulinum toxin A injection in these patients but in upper and lower limb spasticities. Fock et al did a prospective, nonrandomized study in seven patients with a history of TBI secondary to motor vehicle trauma and suffering from spastic equinus ${ }^{24}$. Patients were injected with onabotulinumtoxin A into the heads of the gastrocnemius and soleus muscles only, totaling $300 \mathrm{u}$, and six of the seven patients showed significant improvements in walking speed and stride length at 12 weeks ${ }^{24}$. In this study significant improvements were seen 
in quality of life following intradetrusor injection of incobotulinumtoxinA. $90 \%$ of patients who completed the PGI-I postoperatively noted some degree of improvement within the month after their injections. These findings are in keeping with other studies examining the effects of intradetrusor injection of botulinum toxin. Ginsberg et al showed a significant improvement in impact on quality of life in neurogenic bladder patients, which was not impacted by the initiation of $\mathrm{CIC}^{6}$. Similarly, Nitti et al showed that in idiopathic $\mathrm{OAB}$ patients there were significant improvement in patients' quality of life, even as early as 2 weeks postoperatively ${ }^{5}$. Finally, Chapple et al described significant improvements in the impact on QOL survey and the King's Health Questionnaire within 12 weeks of treatment in idiopathic DO patients in a randomized trial ${ }^{25}$. These significant changes in quality of life are important factors to consider, especially with neurogenic patients who are often managing multiple medical conditions. Perceived effects of treatments by the patients enhance the therapeutic benefits. This positive feedback is encouraging as we seek to expand the use of incobotulinumtoxinA for OAB and UI.

Well established side effects after intradetrusor botulinumtoxin A injections include urinary retention, urinary tract infection, dysuria, and bacteriuria. Less frequent side effects include hematuria and pyrexia.

In the first 12 weeks following treatment, Ginsberg et al described UTI incidence of $28 \%$ in both treatment groups ( $200 \mathrm{u}$ and $300 \mathrm{u}$ ), with hematuria and pyrexia occurring in $5 \%$ or less in both treatment groups ${ }^{6}$. Nitti et al also described adverse events in the first 12 weeks following injection, showing an incidence of $15.5 \%{ }^{5}$. No patients reported adverse effects in this study. This may partly be due to small sample size and short duration of follow-up in this study. However, it is also our practice to treat bacteriuria prior to intradetrusor injection of incobotulinumtoxinA, which may limit the incidence of symptomatic UTI. Overall, the procedure was well tolerated.

One limitation in this study includes the fact that it was a retrospective study. This pilot study has a small sample size and would need to be powered appropriately on a larger scale to draw firmer conclusions. The study population was limited to men because of the site location (VAMC) so generalizability to women should be further explored in larger studies. Future larger placebo-controlled longer-term studies are needed and other populations that should be studied include patients with idiopathic OAB. Other limitations include variations in follow up times between 2-4 weeks. Additionally, no post-injection UDS data was obtained in patients with NDO.

\section{Conclusions}

In this preliminary pilot study of a small cohort of males with neurogenic DO and UI, significant improvements were seen following incobotulinumtoxinA injection in daily frequency, number of incontinence episodes, hours in between CIC, and quality of life scores. Larger scale and long-term studies are required to confirm these results, but initial findings are promising for wider use of this formulation. 


\section{References}

1. Nambiar, A., Lucas, M.: Chapter 4: Guidelines for the diagnosis and treatment of overactive bladder (OAB) and neurogenic detrusor overactivity (NDO). Neurourol Urodyn, 33 Suppl 3: S21, 2014

2. Santos-Silva, A., da Silva, C. M., Cruz, F.: Botulinum toxin treatment for bladder dysfunction. Int J Urol, 20: 956, 2013

3. Mangera, A., Andersson, K. E., Apostolidis, A. et al.: Contemporary management of lower urinary tract disease with botulinum toxin A: a systematic review of 
botox (onabotulinumtoxinA) and dysport (abobotulinumtoxinA). Eur Urol, 60: 784, 2011

4. Mangera, A., Apostolidis, A., Andersson, K. E. et al.: An updated systematic review and statistical comparison of standardised mean outcomes for the use of botulinum toxin in the management of lower urinary tract disorders. Eur Urol, 65: 981,2014

5. Nitti, V. W., Dmochowski, R., Herschorn, S. et al.: OnabotulinumtoxinA for the treatment of patients with overactive bladder and urinary incontinence: results of a phase 3, randomized, placebo controlled trial. J Urol, 189: 2186, 2013

6. Ginsberg, D., Gousse, A., Keppenne, V. et al.: Phase 3 efficacy and tolerability study of onabotulinumtoxinA for urinary incontinence from neurogenic detrusor overactivity. J Urol, 187: 2131, 2012

7. Kennelly, M., Dmochowski, R., Schulte-Baukloh, H. et al.: Efficacy and safety of onabotulinumtoxinA therapy are sustained over 4 years of treatment in patients with neurogenic detrusor overactivity: Final results of a long-term extension study. Neurourol Urodyn, 36: 368, 2017

8. Ravindra, P., Jackson, B. L., Parkinson, R. J.: Botulinum toxin type A for the treatment of non-neurogenic overactive bladder: does using onabotulinumtoxinA $(\operatorname{Botox}((\mathrm{R})))$ or abobotulinumtoxinA (Dysport((R)) ) make a difference? BJU Int, 112: 94,2013

9. Peyronnet, B., Roumiguie, M., Castel-Lacanal, E. et al.: Efficacy and safety of the first and repeated intradetrusor injections of abobotulinum toxin A $750 \mathrm{U}$ for treating neurological detrusor overactivity. World J Urol, 34: 755, 2016

10. Hirst, G. R., Watkins, A. J., Guerrero, K. et al.: Botulinum toxin B is not an effective treatment of refractory overactive bladder. Urology, 69: 69, 2007

11. Walker, T. J., Dayan, S. H.: Comparison and overview of currently available neurotoxins. J Clin Aesthet Dermatol, 7: 31, 2014

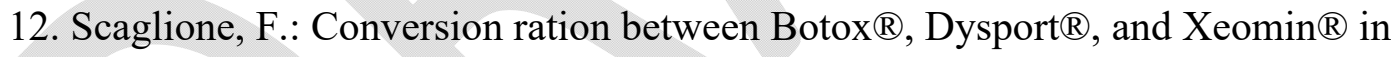
Clinical Practice. Toxins (Basel), 8: 65, 2016

13. Wissel, J., Bensmail, D., Ferreira, J. et al.: Safety and efficacy of incobotulinumA doses up to $800 \mathrm{U}$ in limb spasticity the Tower study. Neurolofy, 88: 1321, 2017

14. Soares, D. J., Dejoseph, L. M., Zuliani, G. F. et al.: Impact of Postreconstitution Room Temperature Storage on the Efficacy of IncobotulinumtoxinA Treatment of Dynamic Lateral Canthus Lines. Dermatol Surg, 41: 712, 2015

15. Jost, W. H., Kohl, A., Brinkmann, S. et al.: Efficacy and tolerability of a botulinum toxin type A free of complexing proteins (NT 201) compared with commercially available botulinum toxin type A (BOTOX) in healthy volunteers. J Neural Transm (Vienna), 112: 905, 2005

16. Scaglione, F.: Conversion Ratio between Botox(R), Dysport(R), and Xeomin(R) in Clinical Practice. Toxins (Basel), 8, 2016

17. Linsenmeyer, T. A.: Use of botulinum toxin in individuals with neurogenic detrusor overactivity: state of the art review. J Spinal Cord Med, 36: 402, 2013

18. Tilden, D., Guarnieri, C.: Cost-Effectiveness of Incobotulinumtoxin-A with Flexible Treatment Intervals Compared to Onabotulinumtoxin-A in the 
Management of Blepharospasm and Cervical Dystonia. Value Health, 19: 145, 2016

19. http://www.xeomin.com/wp-content/uploads/xeomin-full-prescribinginformation.pdf: Prescribing Information for XEOMIN. , 2018

20. Kanovsky, P., Slawek, J., Denes, Z. et al.: Efficacy and safety of treatment with incobotulinum toxin A (botulinum neurotoxin type A free from complexing proteins; NT 201) in post-stroke upper limb spasticity. J Rehabil Med, 43: 486, 2011

21. Conte, A., Giannantoni, A., Gubbiotti, M. et al.: Intradetrusorial Botulinum Toxin in Patients with Multiple Sclerosis: A Neurophysiological Study. Toxins (Basel), 7: 3424,2015

22. Rosier, P., Schaefer, W., Lose, G. et al.: International Continence Society Good Urodynamic Practices and Terms 2016: Urodynamics, uroflowmetry, cystometry, and pressure-flow study. Neurourol Urodyn, 36: 1243, 2017

23. Shumaker, S. A., Wyman, J. F., Uebersax, J. S. et al.: Health-related quality of life measures for women with urinary incontinence: the Incontinence Impact Questionnaire and the Urogenital Distress Inventory. Continence Program in Women (CPW) Research Group. Qual Life Res, 3: 291, 1994

24. Fock, J., Galea, M. P., Stillman, B. C. et al.: Functional outcome following Botulinum toxin A injection to reduce spastic equinus in adults with traumatic brain injury. Brain Inj, 18: 57, 2004

25. Chapple, C., Sievert, K. D., MacDiarmid, S. et al.: OnabotulinumtoxinA 100 U significantly improves all idiopathic overactive bladder symptoms and quality of life in patients with overactive bladder and urinary incontinence: a randomised, double-blind, placebo-controlled trial. Eur Urol, 64: 249, 2013 
Figures and Tables

Fig. 1. Patient Global Impression of Improvement Scores, voiding vs. non-voiding patients.

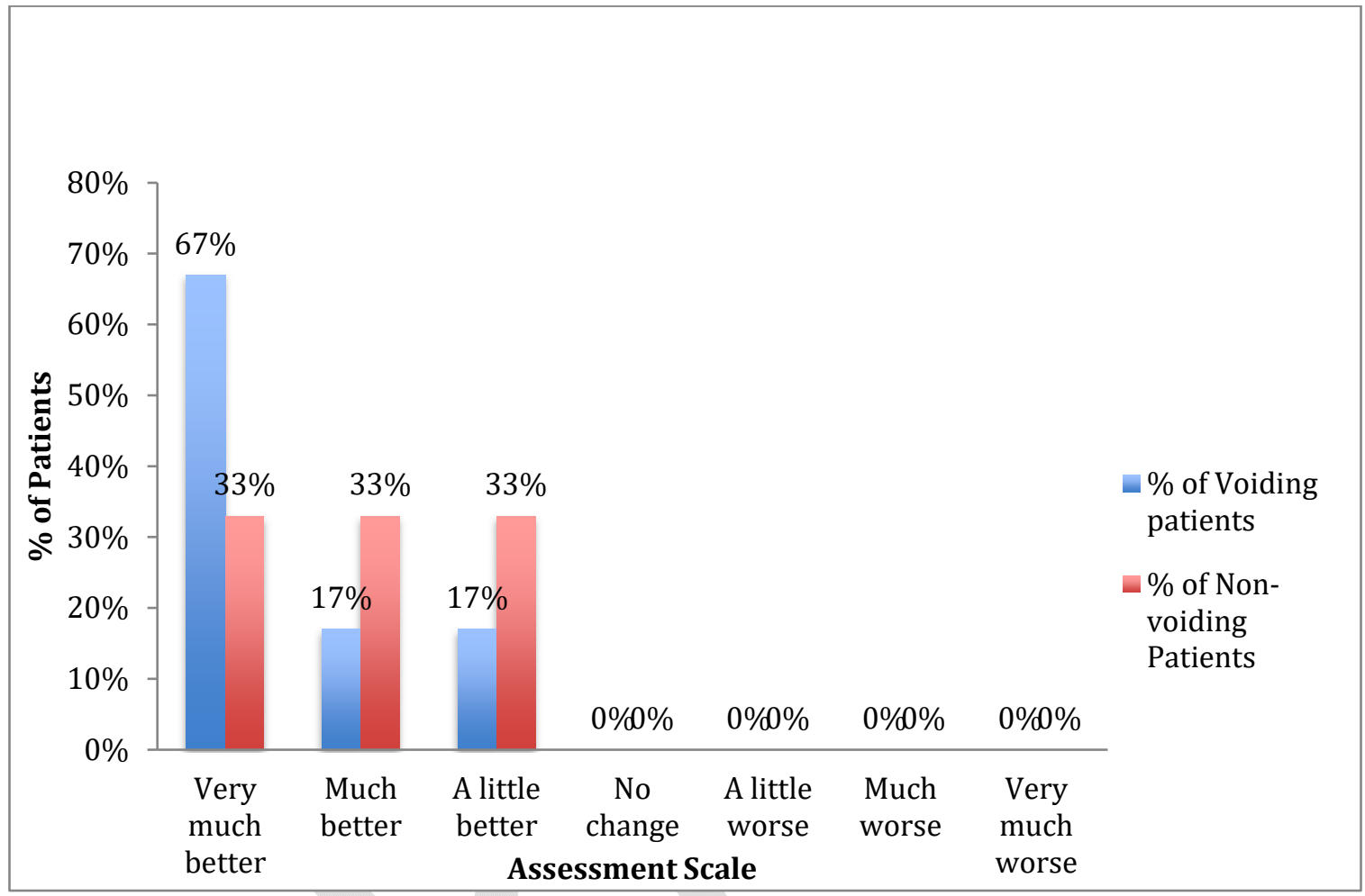

\section{Table 1. Patient demographics}

\begin{tabular}{|l|c|}
\hline Total patients & $17($ all male) \\
\hline Age & $61.2 \pm 15.4(2181)$ \\
\hline Race & Patients, n (\%) \\
\hline White & $14(82 \%)$ \\
\hline African American & $3(18 \%)$ \\
\hline Voiding status & Patients, n (\%) \\
\hline Voiding & $6(35 \%)$ \\
\hline Chronic in-dwelling tube & $6(35 \%)$ \\
\hline CIC & $5(30 \%)$ \\
\hline Neurological diagnosis & Patients, n (\%) \\
\hline SCI & $11(65 \%)$ \\
\hline Cervical SCI & $7(41 \%)$ \\
\hline Thoracic SCI & $4(24 \%)$ \\
\hline MS & $1(6 \%)$ \\
\hline Parkinson's disease & $2(12 \%)$ \\
\hline Stroke & $4(24 \%)$ \\
\hline
\end{tabular}


IncobotulinumtoxinA to treat neurogenic detrusor overactivity

\begin{tabular}{|l|c|}
\hline TBI & $5(29 \%)$ \\
\hline Bladder capacity $(\mathrm{mL})$ & median 215, IQR 200-462.5 \\
\hline Baseline creatinine (mg/dL) & median 0.8, IQR 0.6-1.2 \\
\hline Charlson age comorbidity index & $4.3 \pm 2.3$ (median 4, IQR 2-6.5) \\
\hline Incobotulinumtoxin A injection history & Patients, $\mathbf{n}(\%)$ \\
\hline $\begin{array}{l}\text { Prior onobotulinumtoxin or } \\
\text { incobotulinumtoxin injections before } \\
\text { study period }\end{array}$ & $8(47 \%)$ \\
\hline
\end{tabular}

CIC: clean intermittent catheterization; IQR: interquartile range; MS: multiple sclerosis;

SCI: spinal cord injury; TBI: traumatic brain injury.

\section{Table 2. Outcomes following incobotulinumtoxin A injection in CIC patients}

\begin{tabular}{|c|c|c|}
\hline & $\begin{array}{c}\text { Pre-incobotulinumtoxinA } \\
\text { Median (IQR) }\end{array}$ & $\begin{array}{c}\text { Post-incobotulinumtoxinA } \\
\text { Median (IQR) }\end{array}$ \\
\hline Daily pads & $0(0-6)$ & $0(0-0)$ \\
\hline $\begin{array}{l}\text { \# incontinent episodes } \\
\text { daily }\end{array}$ & $2(1-3)$ & $0(0-0.25)$ \\
\hline Creatinine $(\mathrm{mg} / \mathrm{dL})$ & $0.7(0.6-0.8)$ & $0.5(0.4-0.75)$ \\
\hline CIC volumes (mL) & $400(300-500)$ & $550(400-700)$ \\
\hline Hours in between CIC & $4(3-4)$ & $6(4-6)$ \\
\hline UDI6 scores $($ mean \pm SD) & $31.5 \pm 27.4$ & $8.9 \pm 11.5$ \\
\hline IIQ7 scores (mean \pm AD) & $64.3 \pm 33.3$ & $9.5 \pm 11.2$ \\
\hline $\begin{array}{l}\text { Patients requiring pads, } \mathrm{n} \\
(\%)\end{array}$ & $3(60 \%)$ & $0(0 \%)$ \\
\hline Dry patients, n (\%) & $0(0 \%)$ & $5(0 \%)$ \\
\hline
\end{tabular}

CIC: clean intermittent catheterization; IQR: interquartile range; SD: standard deviation. 


\begin{tabular}{|l|c|c|}
\hline \multicolumn{3}{|c|}{ Table 3. Outcomes following incobotulinumtoxinA injection in voiding patients } \\
\hline & $\begin{array}{c}\text { Pre-incobotulinumtoxinA } \\
\text { Median (IQR) }\end{array}$ & $\begin{array}{c}\text { Post-incobotulinumtoxinA } \\
\text { Median (IQR) }\end{array}$ \\
\hline Daily pads & $7.5(0.75-16.5)$ & $12(0-18)$ \\
\hline Daily frequency & $11(8.5-18)$ & $1(2-10)$ \\
\hline $\begin{array}{l}\text { \# incontinent episodes } \\
\text { daily }\end{array}$ & $2.5(1-5.5)$ & $1.7(1.1-1.9)$ \\
\hline Creatinine & $1.75(1.2-1.9)$ & $35.1 \pm 56.2$ \\
\hline $\begin{array}{l}\text { UDI-6 scores (mean } \pm \\
\text { SD) }\end{array}$ & $36.1 \pm 43.1$ & $33.3 \pm 57.7$ \\
\hline IIQ7 scores (mean \pm SD) & $52.4 \pm 40.4$ & $2(33 \%)$ \\
\hline $\begin{array}{l}\text { Patients requiring pads, } \mathrm{n} \\
(\%)\end{array}$ & $3(50 \%)$ & $2(33 \%)$ \\
\hline Dry patients, $\mathrm{n}(\%)$ & $0(0 \%)$ & $\begin{array}{c}\text { ( } \%) \\
\text { CIC: }\end{array}$ \\
\hline
\end{tabular}

CIC: clean intermittent catheterization; IQR: interquartile range; SD: standard deviation.

Table 4. Pre and post-incobotulinumtoxinA injection outcomes in patients with chronic indwelling tube (Foley catheters and suprapubic catheters)

\begin{tabular}{|l|c|c|}
\hline & $\begin{array}{c}\text { Pre-incobotulinumtoxinA } \\
\text { Median (IQR) }\end{array}$ & $\begin{array}{c}\text { Post-incobotulinumtoxinA } \\
\text { Median (IQR) }\end{array}$ \\
\hline Creatinine mg/dL & $0.9(0.5-1.1)$ & $0.9(0.6-1)$ \\
\hline $\begin{array}{l}\text { IIQ7 mean scores (mean } \pm \\
\text { SD) }\end{array}$ & $39.3 \pm 27.8$ & $1.6 \pm 2.8$ \\
\hline $\begin{array}{l}\text { UDI-6 mean scores (mean } \\
\pm \text { SD) }\end{array}$ & $25 \pm 27.4$ & $8.4 \pm 11.8$ \\
\hline $\begin{array}{l}\text { Patients requiring pads, } \mathrm{n} \\
(\%)\end{array}$ & $1(17 \%)$ & $1(17 \%)$ \\
\hline Dry patients, $\mathrm{n}(\%)$ & $0(0 \%)$ & $2(33 \%)$ \\
\hline
\end{tabular}

CIC: clean intermittent catheterization; IQR: interquartile range; SD: standard deviation. 